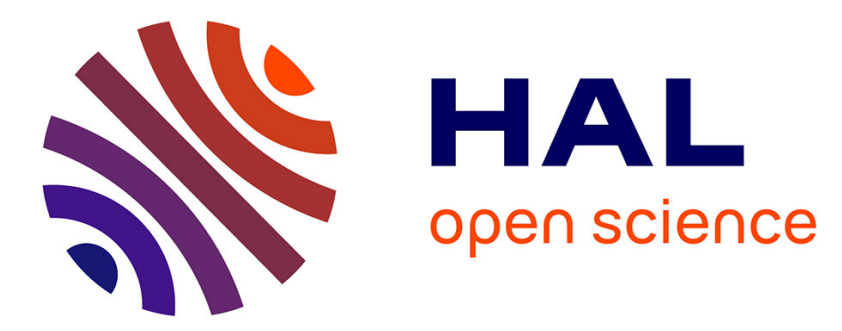

\title{
Cracks and blisters formed close to a silicon wafer surface by He-H co-implantation at low energy
}

Nikolay Cherkashin, Nabil Daghbouj, François-Xavier Darras, M. Fnaiech, Alain Claverie

\section{- To cite this version:}

Nikolay Cherkashin, Nabil Daghbouj, François-Xavier Darras, M. Fnaiech, Alain Claverie. Cracks and blisters formed close to a silicon wafer surface by He-H co-implantation at low energy. Journal of Applied Physics, 2015, 118 (24), pp.245301 - 135308. 10.1063/1.4938108 . hal-01719496

\section{HAL Id: hal-01719496 \\ https://hal.science/hal-01719496}

Submitted on 28 Feb 2018

HAL is a multi-disciplinary open access archive for the deposit and dissemination of scientific research documents, whether they are published or not. The documents may come from teaching and research institutions in France or abroad, or from public or private research centers.
L'archive ouverte pluridisciplinaire HAL, est destinée au dépôt et à la diffusion de documents scientifiques de niveau recherche, publiés ou non, émanant des établissements d'enseignement et de recherche français ou étrangers, des laboratoires publics ou privés. 


\section{Cracks and blisters formed close to a silicon wafer surface by He-H co-implantation at low energy}

N. Cherkashin, N. Daghbouj, F.-X. Darras, M. Fnaiech, and A. Claverie

Citation: Journal of Applied Physics 118, 245301 (2015); doi: 10.1063/1.4938108

View online: https://doi.org/10.1063/1.4938108

View Table of Contents: http://aip.scitation.org/toc/jap/118/24

Published by the American Institute of Physics

\section{Articles you may be interested in}

Effect of the order of $\mathrm{He}^{+}$and $\mathrm{H}^{+}$ion co-implantation on damage generation and thermal evolution of complexes, platelets, and blisters in silicon

Journal of Applied Physics 119, 135308 (2016); 10.1063/1.4945032

Kinetic evolution of blistering in hydrogen-implanted silicon

Applied Physics Letters 103, 031908 (2013); 10.1063/1.4813858

Development of microcracks in hydrogen-implanted silicon substrates

Journal of Applied Physics 114, 123513 (2013); 10.1063/1.4821239

Fracture dynamics in implanted silicon

Applied Physics Letters 107, 092102 (2015); 10.1063/1.4930016

Efficient production of silicon-on-insulator films by co-implantation of $\mathrm{He}^{+}$with $\mathrm{H}^{+}$

Applied Physics Letters 72, 1086 (1998); 10.1063/1.120945

The effect of order and dose of $\mathrm{H}$ and He sequential implantation on defect formation and evolution in silicon Journal of Applied Physics 101, 033506 (2007); 10.1063/1.2432380

\section{Scilight}

Sharp, quick summaries illuminating the latest physics research

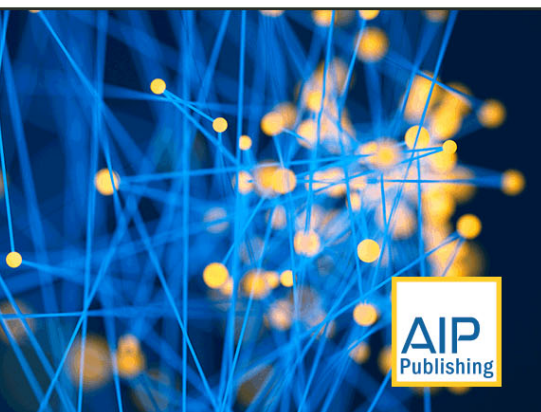




\title{
Cracks and blisters formed close to a silicon wafer surface by He-H co-implantation at low energy
}

\author{
N. Cherkashin, ${ }^{1, a)}$ N. Daghbouj, ${ }^{1,2}$ F.-X. Darras, ${ }^{1}$ M. Fnaiech, ${ }^{2}$ and A. Claverie ${ }^{1}$ \\ ${ }^{1}$ CEMES-CNRS and Université de Toulouse, 29 rue J. Marvig, 31055 Toulouse, France \\ ${ }^{2}$ Faculté des Sciences de Monastir, Université de Monastir, Monastir, Tunisia
}

(Received 21 July 2015; accepted 2 December 2015; published online 22 December 2015)

\begin{abstract}
We have studied the effect of reducing the implantation energy towards low keV values on the areal density of $\mathrm{He}$ and $\mathrm{H}$ atoms stored within populations of blister cavities formed by co-implantation of the same fluence of $\mathrm{He}$ then $\mathrm{H}$ ions into $\mathrm{Si}(001)$ wafers and annealing. Using a variety of experimental techniques, we have measured blister heights and depth from the surface, diameter, areal density of the cracks from which they originate as functions of implantation energy and fluence. We show that there is a direct correlation between the diameters of the cracks and the heights of the associated blisters. This correlation only depends on the implantation energy, i.e., only on the depth at which the cracks are located. Using finite element method modeling, we infer the pressure inside the blister cavities from the elastic deformations they generate, i.e., from the height of the blisters. From this, we demonstrate that the gas pressure within a blister only depends on the diameter of the associated crack and not on its depth position and derive an analytical expression relating these parameters. Relating the pressure inside a blister to the respective concentrations of gas molecules it contains, we deduce the areal densities of $\mathrm{He}$ and $\mathrm{H}$ atoms contained within the populations of blisters. After low-energy implantations $\left(8 \mathrm{keV} \mathrm{He}^{+}, 3 \mathrm{keV} \mathrm{H}^{+}\right)$, all the implanted $\mathrm{He}$ and $\mathrm{H}$ atoms contribute to the formation of the blisters. There is no measurable exo-diffusion of any of the implanted gases, in contrast to what was assumed at the state of the art to explain the failure of the Smart-Cut technology when using very low energy ion implantation for the fabrication of ultra-thin layers. Alternative explanations must be investigated. (C) 2015 AIP Publishing LLC.

[http://dx.doi.org/10.1063/1.4938108]
\end{abstract}

\section{INTRODUCTION}

Hydrogen implantation in Si followed by wafer bonding and annealing allows for slicing and the transfer of relatively thick (i.e., $>200 \mathrm{~nm}$ ) Si layers from a donor substrate to a host material. The Smart $\mathrm{Cut}^{\mathrm{TM}}$ technology, based on this principle, ${ }^{1}$ is used to fabricate Silicon-On-Insulator (SOI) substrates, the starting material of many electronic and photovoltaic modern devices. ${ }^{2,3}$ After implantation and during annealing, silicon vacancies and hydrogen atoms precipitate into the form of platelets of nanometer dimensions that progressively grow by Ostwald ripening. ${ }^{4,5}$ When the concentration of implanted ions is sufficiently large, the platelet population evolves during annealing with the eventual formation of micro-cracks. ${ }^{5}$ These micro-cracks result from the mechanical coalescence of groups of platelets which are sufficiently close to each other to allow an efficient overlap of the stress fields that they generate. ${ }^{6}$ The further thermal evolution of these highly pressurized cracks leads to the fracture phenomenon at the basis of the Smart Cut technology.

There is a strong technological demand to find and develop a method for transferring extremely thin (i.e., $<50 \mathrm{~nm}$ ) layers. ${ }^{7}$ In principle, such an objective could be simply achieved by locating the fracture front closer to the wafer surface, i.e., by implanting $\mathrm{H}^{+}$at very low energy. However, the conditions required to efficiently co-precipitate $\mathrm{H}$ and

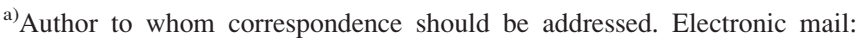
nikolay.cherkashin@cemes.fr
}

vacancies and to form micro-cracks so close to the free surface of a $\mathrm{Si}$ wafer have been found to differ from that of high energy ion implantation. ${ }^{8,9}$ For example, while blistering may be observed after high energy ion implantation at a large enough fluence, blistering is not observed after the implantation of the same fluence at low energy. This characteristic was attributed to the premature desorption of $\mathrm{H}_{2}$ during annealing. ${ }^{8}$ Moreover, the blistering efficiency depends on the amount of the damage produced during ion implantation because it affects the microstructure of the implanted samples. This microstructure depends on a fluence and energy of implanted ions and depends on whether $\mathrm{H}$ or $\mathrm{He}$ are implanted alone or co-implanted. ${ }^{10,11}$ Hydrogen and helium co-implantation, routinely used to reduce the total fluence necessary to transfer thick layers when implanted in quasiequal proportion, ${ }^{12}$ was found to require a higher fluence of $\mathrm{H}$ than He when implanted at low energies. ${ }^{8,9}$ Again the exodiffusion of $\mathrm{H}$ or $\mathrm{He}$ atoms from the implanted region was hypothesized to be responsible for that characteristic.

The optimization of the Smart Cut process using very low energy ion implantation would require a much better understanding of the formation of micro-cracks close to a wafer surface with respect to the state of the art. In particular, the evolution of implanted $\mathrm{H}$ and $\mathrm{He}$ during annealing in relation to the formation of cracks and blisters should be better understood. Currently, no experimental technique allows the direct measurement of the respective gas concentrations of $\mathrm{H}_{2}$ and $\mathrm{He}$ within highly pressurized micro-crack cavities. 
So far, only one report, focused on the mechanical cleavage of the implanted region through the platelets and microcracks, has been used to study high fluence $\mathrm{H}^{+}$implantation in $\mathrm{Si}(001)$ at high energy. ${ }^{13}$

For these reasons, in this work, we study the effect of reducing the ion energies during both $\mathrm{H}$ and $\mathrm{He}$ implantations on the formation and development of micro-cracks. To this purpose, we experimentally study the blisters that are formed when a stiffener, usually another wafer, is not bonded to the implanted wafer after implantation and before annealing. Under such conditions, fracture does not occur and instead blisters appear at the surface of the wafer. A crack is an object created by the coalescence of a group of platelets. The shape of such a gas-filled cavity (crack cavity) can be approximated by an oblate spheroid with a minor semi-axis of subnanometer length. The gas, which is under high pressure, acts on the internal surfaces of the crack inducing stress in the matrix. In absence of a stiffener, and following the crack formation, the stress in the matrix is partially relaxed through the elastic relaxation of the crystal, close to the surface of the wafer. This phenomenon results in the formation of a blister that originates from a gas-filled cavity embedded in the wafer (blister cavity). The height of the blister is defined as the distance from an undistorted, or flat, surface to its apex. By comparing experimental results with Finite Element Method (FEM) modeling results, we deduce their internal pressure and the fraction of the implanted fluence used to pressurize them. We show that even when implanted at very low energy, $\mathrm{H}$ and $\mathrm{He}$ atoms do not exo-diffuse out of the implanted region and that contrary to what is generally assumed, the fraction of the implanted fluence used to pressurize blister cavities actually increases at such energies. The failure of the conventional Smart Cut technology at such low energies cannot be ascribed to the exo-diffusion of the implanted gases in the vicinity of the wafer surface.

\section{EXPERIMENTAL DETAILS AND METHODOLOGY}

(001) Si wafers covered by a $25 \mathrm{~nm}$-thick thermally grown $\mathrm{SiO}_{2}$ layer were co-implanted at room temperature by $\mathrm{He}^{+}$ then $\mathrm{H}^{+}$ions at three different couples of energies, $18 \mathrm{keV}$ $\mathrm{He}^{+}$and $10 \mathrm{keV} \mathrm{H}^{+}, 12 \mathrm{keV} \mathrm{He}^{+}$and $6 \mathrm{keV} \mathrm{H}^{+}, 8 \mathrm{keV} \mathrm{He}^{+}$ and $3 \mathrm{keV} \mathrm{H}^{+}$(thereafter referenced as "high," "medium," or "low" energies implanted samples, respectively). These wafers were implanted with the same fluences of $\mathrm{He}$ and $\mathrm{H}$ ions, each one of $0.6 \times 10^{16} \mathrm{~cm}^{-2}$ or $1.2 \times 10^{16} \mathrm{~cm}^{-2}$ (thereafter referenced as "low" and "high" fluence samples, respectively). These couples of $\mathrm{He}^{+}$and $\mathrm{H}^{+}$implantation energies were selected such that the $\mathrm{He}^{+}$peak concentration is always located deeper and at approximately $50 \mathrm{~nm}$ from the $\mathrm{H}^{+}$peak concentration (see Fig. 1), similarly to what is done in the Smart Cut process but using much higher energies (typically in the $30-50 \mathrm{keV}$ range) for transferring thick layers. The samples were then annealed at $550^{\circ} \mathrm{C}$ for 30 min under nitrogen gas in a conventional furnace.

Cross-sectional transmission electron microscopy (TEM) under out-of-Bragg and out-of-focus conditions was used to image platelets and micro-cracks. ${ }^{14,15}$ Plan view scanning electron microscopy (SEM) in the backscattered

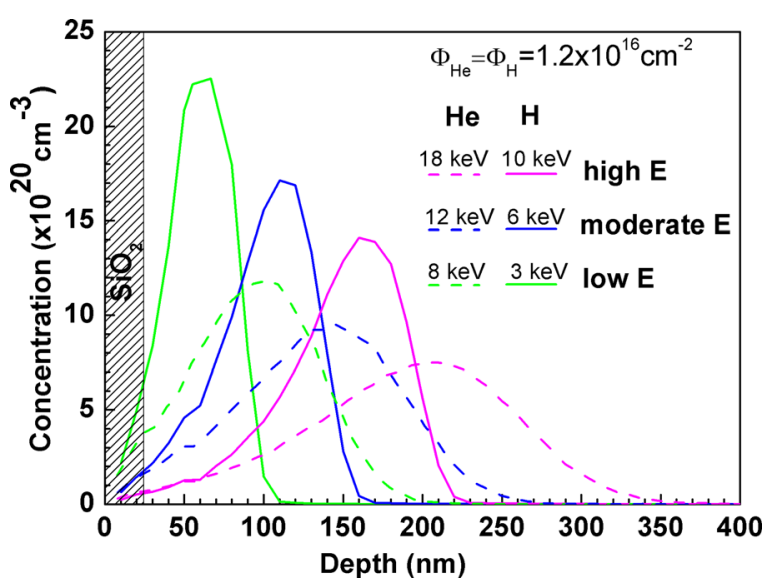

FIG. 1. H (solid lines) and He (dashed lines) implantation profiles calculated by SRIM for the "high fluence" samples.

electron imaging mode was used to observe the blister cavities, while the morphology of the blisters was imaged by Atomic Force Microscopy (AFM) in non-contact mode. FEM modeling of the deformation generated by gas filled blister cavities in the vicinity of a free surface was implemented using COMSOL Multiphysics 4.3.

\section{EXPERIMENTAL RESULTS}

Figure 2 shows the defects formed after annealing of the wafers co-implanted at high, moderate, and low energies (from top to bottom) and for the two fluences investigated here. Both

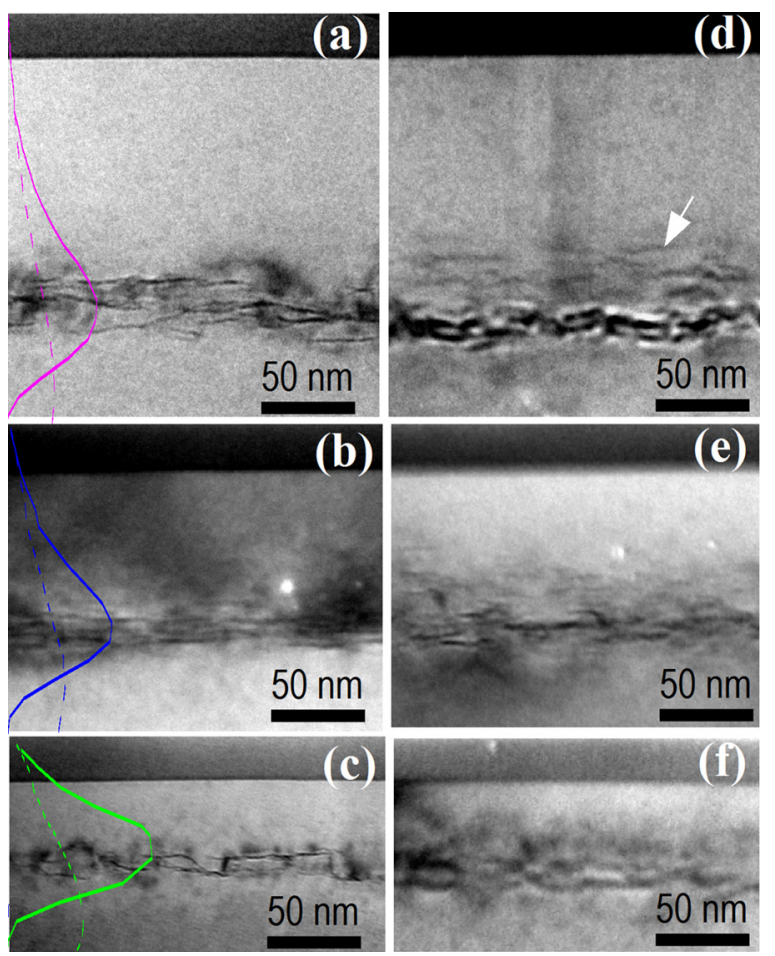

FIG. 2. Bright-field off-Bragg defocused cross-sectional (110) TEM images of the different implanted samples after $550^{\circ} \mathrm{C}, 30 \mathrm{~min}$ annealing. From top to bottom, samples implanted at high, moderate, and low energy. Left column, samples implanted at low fluence (a), (b), and (c) and right column, at high fluence (d), (e), and (f). The inserted line profiles show the $\mathrm{H}$ (solid lines) and the He (dashed lines) depth distributions obtained by SRIM. The arrow in (d) shows a platelet. 
the platelets and the micro-cracks are plate-like cavities filled with hydrogen gas. Under off-Bragg overfocused imaging conditions, the micro-cracks are seen in the cross-sectional TEM images, such as Fig. 2, as zigzag lines while the platelets are seen as straight segments (see Refs. 14 and 15). As the energy decreases, the depth position (distance towards the wafer surface) of the cracks decreases from $140 \mathrm{~nm}$, to $100 \mathrm{~nm}$ and $60 \mathrm{~nm}$, as approximately predicted for the $\mathrm{H}$ peak positions by the SRIM code. ${ }^{16}$

In the samples implanted at low energy, only large micro-cracks are observed. In contrast, in the sample implanted at moderate and high energies, the micro-cracks are surrounded by platelets. The depth distribution of platelets is wider for the high fluence samples. Both the platelets and micro-cracks are found in the region where the hydrogen concentration is maximum (and not $\mathrm{He}$ ) confirming that the nucleation and growth of these defects result from the initial precipitation of hydrogen complexes followed by the diffusion and injection of He molecules towards these precursors. ${ }^{6}$

Figure 3 shows a set of plan view SEM images of the same samples arranged as in Fig. 2. In the backscattered electron imaging mode, the blisters are easily visible as their contrast arises from the gas filled cavity they host. They have a quasi-circular shape.

Their sizes and densities dramatically depend on both the implantation energies and fluences. As the blister cavities are located closer and closer to the surface (i.e., when decreasing the implantation energy), they become smaller but appear in higher density. Moreover, at a fixed implantation energy, when increasing the fluence, the blister cavity diameters
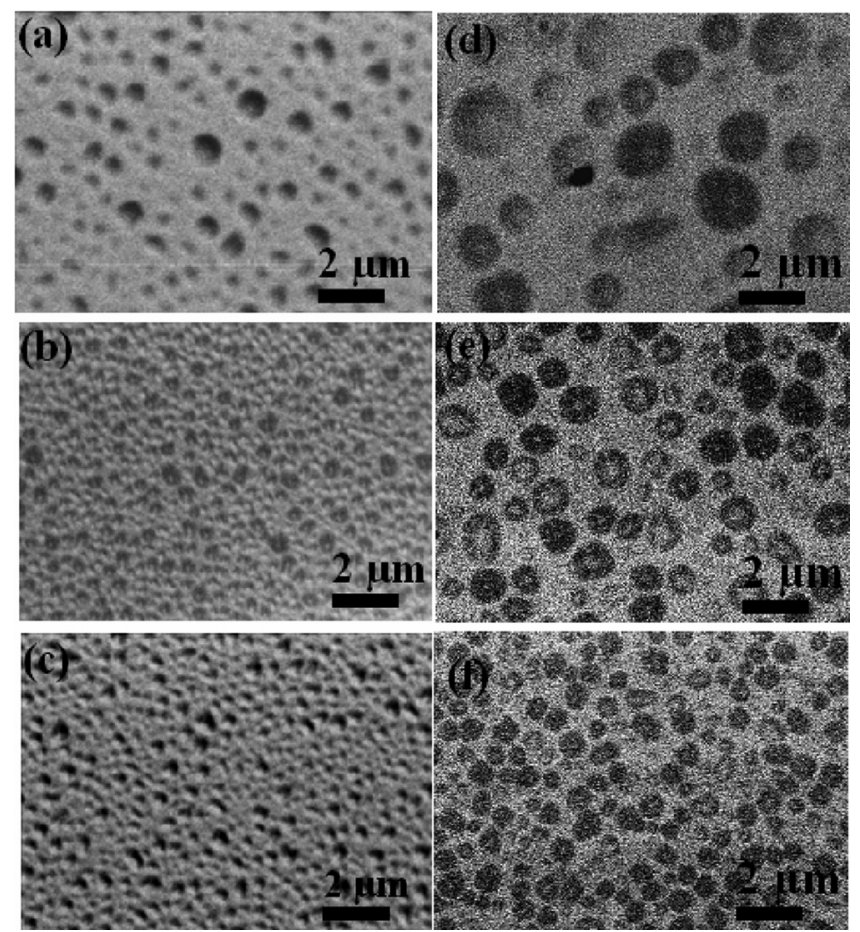

FIG. 3. SEM images (backscattered electrons) of the different implanted samples after $550^{\circ} \mathrm{C}, 30 \mathrm{~min}$ annealing. From top to bottom, samples implanted at high, moderate, and low energy. Left column, samples implanted at low fluence (a), (b), and (c) and right column, at high fluence (d), (e), and (f). increase while their density decreases. The result of the statistical analysis of such images is shown in Fig. 4(a).

The surface fraction occupied by the blister cavities can be calculated as $S f r=\sum_{i} \pi r_{i}^{2} / S$, where $r_{i}$ is the radius of the $i$-th blister cavity, and the summation is made over all the blister cavities found in the area $\mathrm{S}$. The surface fractions occupied by the blister cavities as a function of the implantation energy and for the two fluences of interest are plotted in Fig. 4(b). Figure 4(b) evidences that, whatever the fluence, the surface fraction occupied by the blister cavities is independent of the implantation energy but it slightly increases from $60 \%$ to $70 \%$ when doubling the fluence from $0.6 \times 10^{16} \mathrm{~cm}^{-2}$ to $1.2 \times 10^{16} \mathrm{~cm}^{-2}$.

Figure 5 shows the AFM images of the surfaces of the implanted and annealed wafers using the same color scale for each implantation fluence.

White spots reveal protuberances due to the presence of blisters deforming the surface. The brighter the spot is, the higher the corresponding blister. Obviously, the height of these blisters depends on both the implantation energy and the fluence. Visually, on average, the spots seen in Figs. 5(a) and 5(d) are less bright than the spots seen in Figs. 5(c) and 5(f), respectively. On average, the height increases when the implantation energy decreases, i.e., when the associated cracks are located closer to the surface. Moreover, they also increase when the fluence is doubled. SEM and AFM images of the same regions of the samples unambiguously show the one-to-one relation between blister cavity diameters and

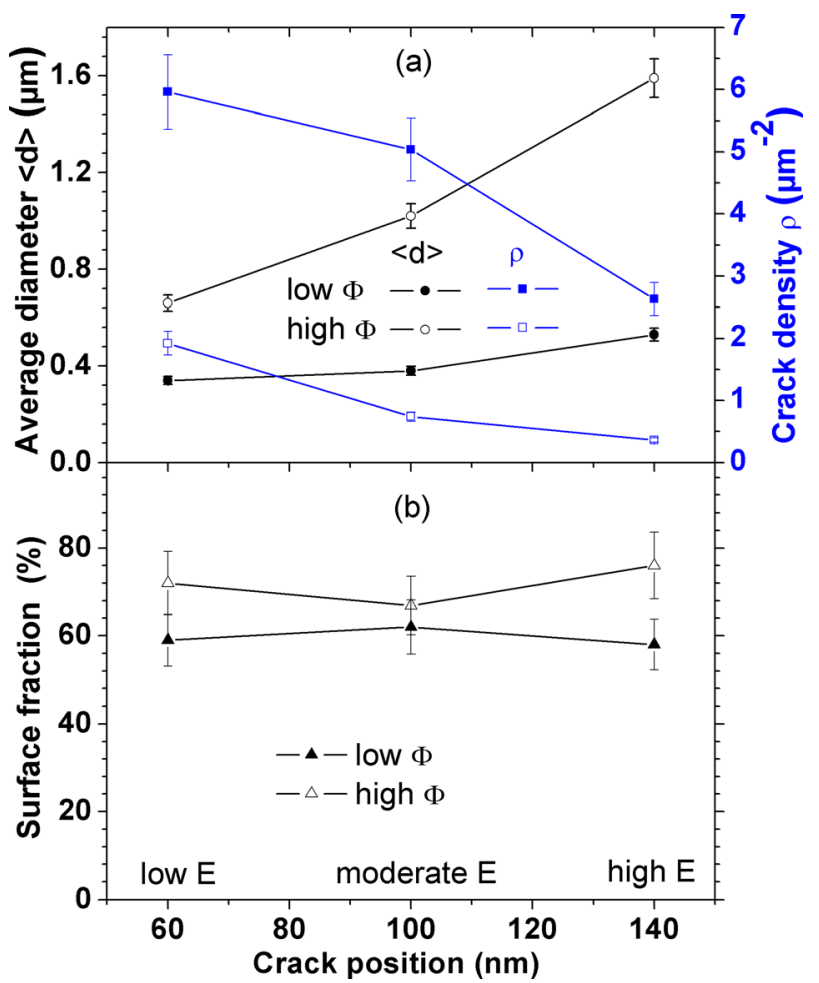

FIG. 4. (a) Average diameter (black symbols) and density (blue symbols) of the blister cavities as a function of their depth position (implantation energy), after low fluence (solid symbols) and high fluence (open symbols) implantations; (b) surface fraction occupied by the blister cavities as a function of their depth position (implantation energy) after low fluence (solid symbols) and high fluence (open symbols) implantations. 

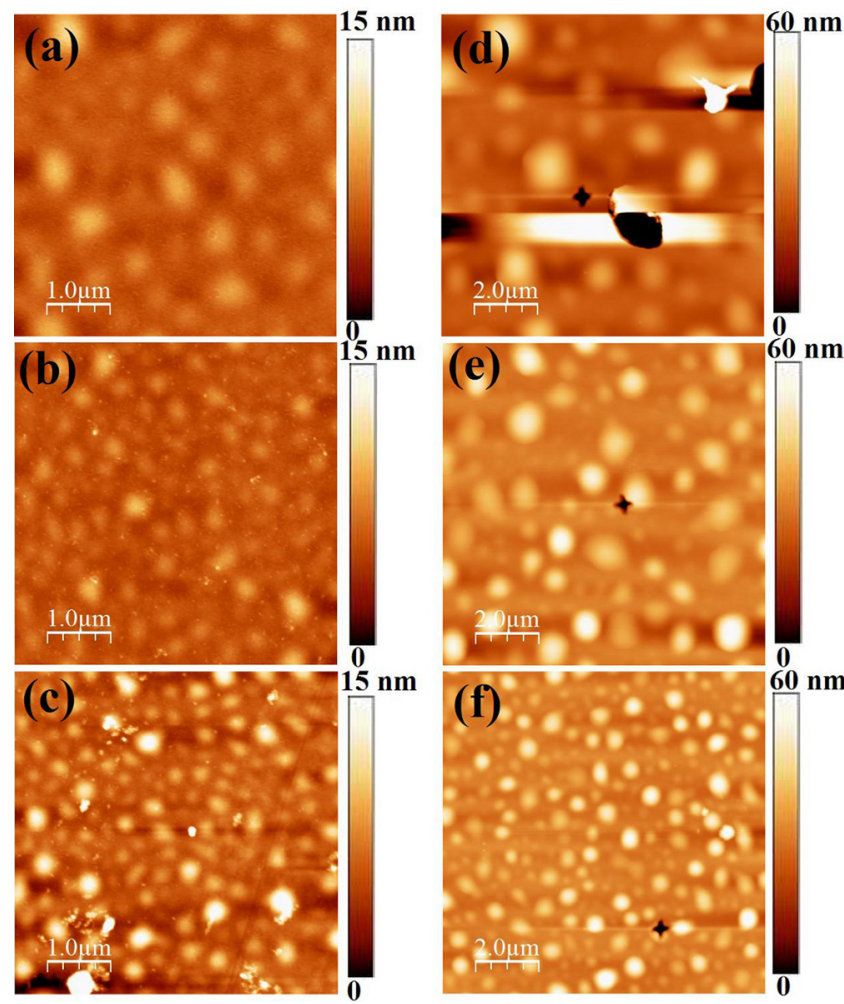

FIG. 5. AFM images of the surfaces implanted at low (a), (b), and (c) and high fluences (d), (e), and (f). The implantation energy decreases from the top to the bottom, i.e., (a) and (d) high energy, (b) and (e) moderate energy, and (c) and (f) low energy.

blister heights. From the analysis of such AFM and SEM images, we have plotted in Fig. 6 the blister heights measured by AFM as a function of blister cavity diameters measured by SEM.

This graph shows that there is a direct correlation between the diameter of a blister cavity and the blister height. The larger the blister cavity diameter is, the higher the corresponding blister. This relation depends only on the

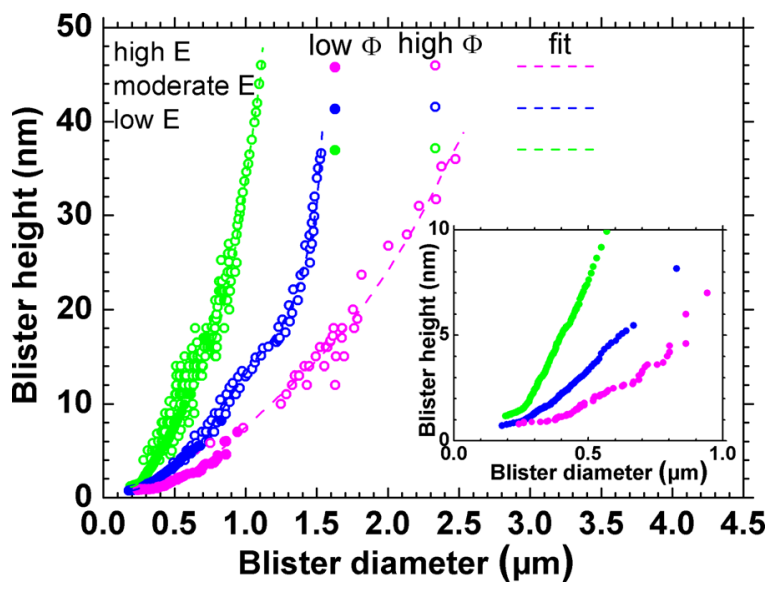

FIG. 6. Blister height as a function of blister cavity diameter for the high energy (violet symbols), moderate energy (blue symbols), and low energy (green symbols) implanted samples. The solid and open symbols correspond to low and high fluence samples, respectively. The dashed lines are fits to the experimental data giving a one-to-one relation between blister diameter and blister height. The data for the low fluence samples are shown in inset for a better visualization. implantation energy (and not on the implanted fluence), i.e., on the depth location of the crack. A crack of a given diameter gives rise to a blister of larger height when located closer to the surface.

\section{FINITE ELEMENT MODELLING}

FEM was used to infer the gas pressure inside a blister of a given diameter-originating from a crack located at a certain depth-from the height of the blister. ${ }^{17-19}$ A microcrack results from the mechanical coalescence of groups of platelets, which are sufficiently close to each other and that can be distributed at slightly different depths from the wafer surface. In any case, the roughness of the internal surfaces defining a crack is negligibly small when compared to its diameter, and thus a micro-crack can be considered to lie on a single habit plane. In our model, a crack is initially described as a cavity of oblate spheroidal shape characterized by a major and a minor axis (see Fig. 7(a)). The length of the minor axis $b$ equals the crack thickness, while the length of the major axis $a$ is the crack diameter.

The crack was embedded within a semi-infinite (001) Si substrate covered by a $25 \mathrm{~nm}$-thick oxide layer and located at a depth $h$ from the wafer surface. The wafer surface was initially set unstressed in the vertical direction and free to move in any direction. The substrate was rigidly fixed at the bottom of the model structure (the box). The vertical "walls" limiting the substrate at the edges of the box were only allowed to move vertically, and the crack was set at sufficiently large distances from any of the box walls so as not to impact the calculated displacement and strain fields. The boundary conditions at the internal surface of the crack were adjusted so that a pre-chosen value of pressure inside the blister cavity formed from the crack, after elastic relaxation, was obtained. 3D maps of the vertical displacement fields

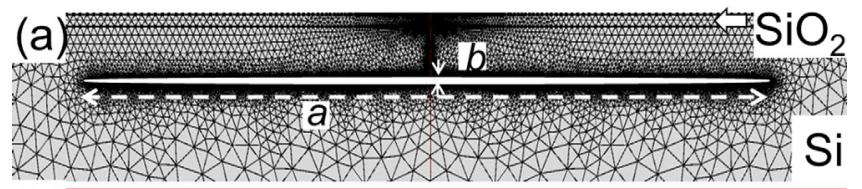

(b)

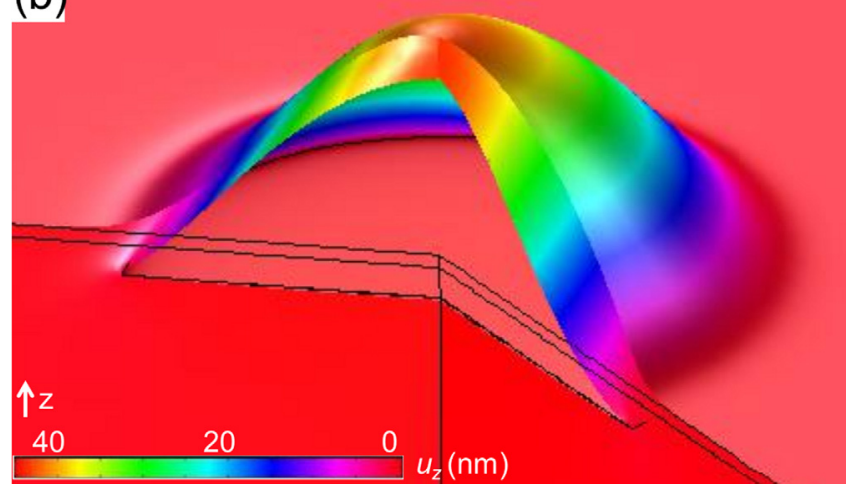

FIG. 7. FEM modeling (in a cylindrical coordinate system) of a blister cavity subjected to an internal gas pressure of $0.12 \mathrm{GPa}$ and formed from an oblate spheroidal crack having a diameter of $1.1 \mu \mathrm{m}$, a thickness of $1 \mathrm{~nm}$, and located at a distance of $60 \mathrm{~nm}$ from the wafer surface. (a) Initial crack configuration with major axis $a$ and minor axis $b$. (b) Calculated displacement field $u_{z}$ in the direction perpendicular to the wafer surface. The vertical dimension was amplified by a factor of 5 for a better visualization. 
induced by such blister cavities could then be plotted (Fig. 7(b)). Note that the diameters of a crack and a blister cavity remain the same, but the shape of the cavity changes to a blister shape through the asymmetrical increase of its upper minor semi-axis length, from $0.5 \mathrm{~nm}$ to approximately the height of the blister.

Thus, given a particular diameter, depth position of a crack, and height of a blister developed from the crack, we can infer the blister cavity volume and the internal pressure of the gas which fills it from FEM simulations (Fig. 7(b)).

\section{DISCUSSION}

Experimentally, we have shown that once the depth position of a crack is fixed, the height of the blister it generates is only a function of its diameter (Fig. 6). Since from this oneto-one relation provided experimentally (Fig. 6, dashed lines), we can retrieve the internal pressure inside the corresponding blister cavity using FEM (Fig. 7(b)), we have plotted in Fig. 8 the variations of the internal pressure inside a blister cavity as a function of its diameter, for the implanted samples explored in this work at three energies and two fluences.

This figure shows surprisingly that the gas pressure inside a blister does not depend on the depth-position of the corresponding crack it originated nor from the implanted fluence but only on its diameter. Although blisters originating from cracks of the same diameter but located at different depths develop different volumes, the pressure and, thus, the gas concentration inside these cavities are the same. This result can be extrapolated to very large depths. Cracks of the same diameter but embedded within an infinite medium, i.e., not generating blisters visible at the surface, preserve the oblate spheroidal shape they established when formed by the coalescence of platelets. Such plate-like cracks are similar in structure and characteristics to platelets, and the pressure inside them can be analytically related to the deformation field it generates in the surrounding matrix via the

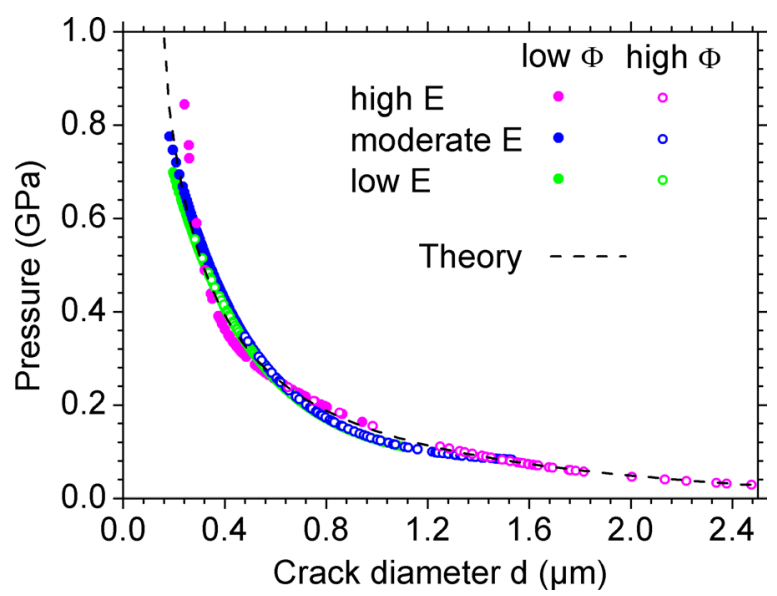

FIG. 8. Pressure inside a blister cavity as a function of its diameter deduced from FEM using the one to one relation between the blister height and the blister cavity diameter provided experimentally (Fig. 6, dashed lines). Compilation of results corresponding to high energy (violet symbols), moderate energy (blue symbols), and low energy (green symbols) implanted samples at low and high fluences. The theoretical dependence of the pressure inside a crack embedded within an infinite medium as a function of its diameter is shown in black dashed line. introduction of an effective or pseudo Burgers vector, ${ }^{18}$ as it is commonly done for platelets

$$
P(d)=\frac{\mu b}{\pi(1-\nu)(d / 2)}(\ln (24(d / 2) / b)-1),
$$

where $b$ is the pseudo-Burgers vector of a plate-like crack of diameter $d, \mu=70.71 \mathrm{GPa}$ the shear modulus of silicon, and $\nu=0.2335$ the Poisson coefficient of silicon. This equation allows the pressure inside a blister cavity located close to the wafer surface to be associated with the pressure inside a plate-like crack, embedded within an infinite medium, of the same diameter and with the same gas concentration. The amplitude of the Burgers vector of such plate-like crack should be different from that of a hydrogen platelet estimated of $3 \AA$ in Ref. 21 and of $6 \AA$ in Ref. 20. A hydrogen platelet is typically pressured to dozen of $\mathrm{GPa}^{20,21}$ At this range of pressures, the gas concentration within a platelet and therefore its Burgers vector are quasi-independent of pressure and, thus, of platelet diameter. ${ }^{20}$ The pressures inside the blister cavities that we typically observe are much smaller. Indeed, the internal pressure inside a small blister cavity may be high, $0.85 \mathrm{GPa}$ for a blister of about $0.2 \mu \mathrm{m}$ in diameter, and decreases when its diameter increases, down to $0.03 \mathrm{GPa}$ for large blisters of about $2.5 \mu \mathrm{m}$ in diameter (Fig. 8). Thus, one would expect that when this pressure decreases, i.e., when the diameter of a blister cavity increases, the Burgers vector of the associated plate-like crack decreases.

For this reason, the pressure in a blister cavity of the given diameter (Fig. 8) could be very well fitted by Eq. (1) applied to a plate-like crack, embedded in an infinite medium, of the same diameter and with the same gas concentration, using a relation such as $b(d)=(3.5-d) 10^{-4}$ (where both $d$ and $b(d)$ are expressed in $\mu \mathrm{m}$ ) (Fig. 8, black dashed line). The Burgers vector (at room temperature) of small cracks may be rather high, of $3.3 \AA$ for a crack of about $0.2 \mu \mathrm{m}$ in diameter, and decreases when its diameter increases, down to $1 \AA$ for large cracks of about $2.5 \mu \mathrm{m}$ in diameter. Such variations of the effective Burgers vector reflect the decrease of the gas concentration when the diameter of the crack increases.

Since the blister cavities are filled by helium and/or $\mathrm{H}_{2}$ molecules, it is tempting to estimate the concentration of molecules they contain from their internal pressure. Given the range of pressures found in the blister cavities, the ideal gas law, $c=P / k T$ (where $\mathrm{c}$ is the molecule concentration, $\mathrm{P}$ is the pressure, $\mathrm{k}$ is the Boltzmann constant, and $\mathrm{T}$ is the temperature), is of course not valid. Instead, we have used the experimental data reported in Refs. 22 and 23 (obtained at room temperature) relating the molecule concentrations $C_{H e}$ and $C_{H_{2}}$ to the gas pressure (Fig. 9).

This graph shows that, at high pressure and for a given concentration, hydrogen molecules are more efficient than He atoms to generate pressure. A given (high) pressure can be obtained either by a minimum molar concentration of hydrogen molecules, a maximum concentration of He atoms, or an intermediate concentration of a mixture of both gases.

The total concentration of molecules $C_{\mathrm{HeH}_{2}}\left(P_{i}\left(d_{i}\right)\right)$ $=x C_{H e}\left(P_{i}\left(d_{i}\right)\right)+(1-x) C_{H_{2}}\left(P_{i}\left(d_{i}\right)\right)$ contained within a 


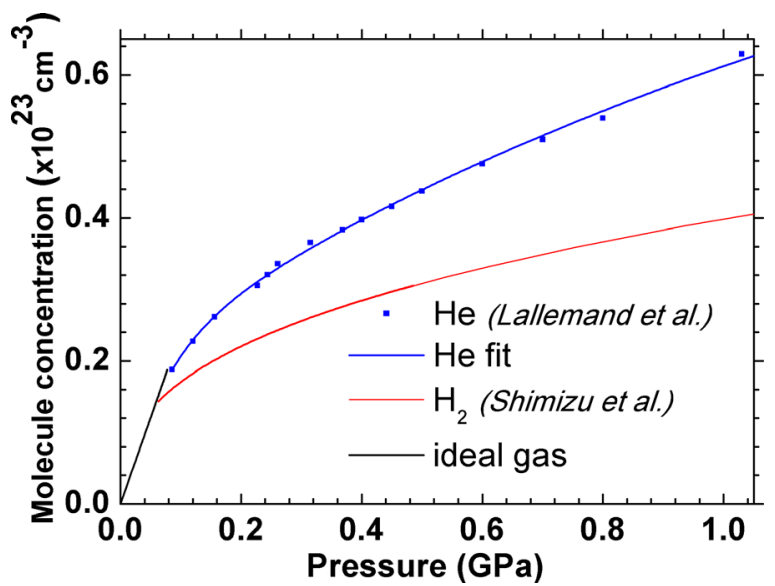

FIG. 9. Relation between concentrations of $\mathrm{He}$ (blue symbols) and $\mathrm{H}_{2}$ (red line) molecules and gas pressure, measured at room temperature (from Refs. 22 and 23). The black line shows this relation for an ideal gas.

blister cavity "i" of diameter $d_{i}$ and volume $V_{i}$ can be obtained by combining the data from Figs. 8 and 9, provided the respective molar fractions $x$ and $(1-x)$ of the two gases are known.

The areal density of molecules, i.e., the number of molecules contained within the blisters and found within a surface area $\mathrm{S}$, is given by

$$
\rho_{\mathrm{HeH}_{2}}=\sum_{i} C_{\mathrm{HeH}_{2}}\left(P_{i}\left(d_{i}\right)\right) V_{i} / S
$$

with $\rho_{\mathrm{HeH}_{2}}=x \rho_{\mathrm{He}}+(1-x) \rho_{\mathrm{H}_{2}}$.

In Figure 10, we have plotted, using blue or red solid squares, respectively, the areal density of molecules that would be needed to generate the observed blisters using exclusively $\mathrm{He}$ or $\mathrm{H}_{2}$ molecules, and this for the 3 different crack depth locations studied in this work. The density needed for any mixture of both gases to generate the same blisters would lie between these two extreme bounds.

At this point, one should compare these areal densities to the implanted fluences. First, this is done in Fig. 10, in blue open squares for He. From this graph, it is already obvious that the blister cavities formed by low and intermediate ion energy implants, at depths of $60 \mathrm{~nm}$ and $140 \mathrm{~nm}$, cannot be pressurized by only $\mathrm{He}$ molecules, since the implanted fluences are smaller than the areal densities of $\mathrm{He}$ molecules required to form the observed blisters.

For hydrogen, the situation is more complex. Since the cracks and their precursors, namely, the platelets, are exactly located at the depth where the concentration of the implanted hydrogen was maximum, and not on the initial He implanted profile, no doubt that $\mathrm{H}$ atoms or molecules are involved in their formation and that they finally are to be found within the blister cavities.

Currently, it is known that platelets and cracks offer large internal (001) surfaces hosting large concentrations of dangling bonds which hydrogen atoms passivate, thus minimizing their energy. ${ }^{24}$ The density of dangling bonds provided by these (001) planes equals $\gamma=4 / a_{S i}^{2}$, where $a_{S i}$ is the lattice parameter of silicon. Each dangling bond is passivated by one hydrogen atom. Thus, the total number of $\mathrm{H}_{2}$ molecules per surface used to passivate the internal

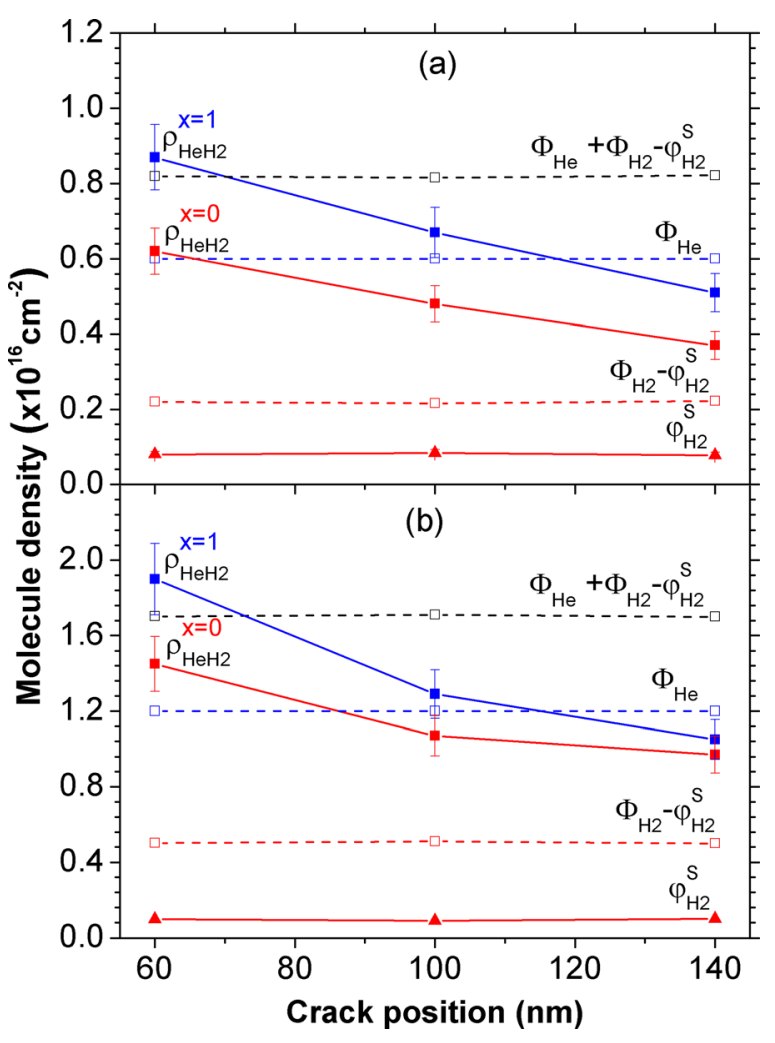

FIG. 10. Density of molecules pressurizing blisters as a function of crack depth position (implantation energy) shown for low fluence (a) and high fluence (b) samples. Blue and red solid squares correspond to the upper and lower limits of molecule density of a mixture of $\mathrm{He}$ and $\mathrm{H}_{2}$ molecules $\rho_{\mathrm{HeH}_{2}}$ calculated for $\mathrm{x}=0$ (red solid squares) and $\mathrm{x}=1$ (blue solid squares). Red solid triangles correspond to a density of $\mathrm{H}_{2}$ molecules passivating the internal surfaces of blister cavities. Open symbols correspond to helium and/or $\mathrm{H}_{2}$ molecule densities available from the implanted fluences for pressuring blisters.

surfaces of the blister cavities found within the area $S$ is given by

$$
\varphi_{H_{2}}^{\text {int.surf }}=\sum_{i} \frac{\gamma S_{i}}{2 S} \cong \sum_{i} \frac{\pi d_{i}^{2}}{a_{S i}^{2} S} .
$$

The values of $\varphi_{\mathrm{H}_{2}}^{\text {int.surf }}$ are also plotted in Fig. 10 (red solid triangles) for low, moderate, and high energy implanted samples. These values, proportional to the surface fraction occupied by the blister cavities (Fig. 4(b)), are quasi-independent of the implantation energy and equal approximately 0.08 $\times 10^{16} \mathrm{~cm}^{-2}$ and $0.1 \times 10^{16} \mathrm{~cm}^{-2}$ after low and high fluence ion implantations, respectively. Thus, in principle, the rest of the hydrogen fluence $\Phi_{H_{2}}-\varphi_{H_{2}}^{\text {int.surf }}\left(\Phi_{H_{2}}=\Phi_{H} / 2\right)$, i.e., more than $80 \%$ of $\Phi_{H}$, is available to pressurize the blister cavities. These values are also plotted in Fig. 10 (red open squares). Finally, the total density of $\mathrm{He}$ atoms and $\mathrm{H}_{2}$ molecules that is available to pressurize the blister cavities is $\Phi_{H_{e}}+\Phi_{H_{2}}-\varphi_{H_{2}}^{\text {int.surf }}$, and these values are plotted in Fig. 10 for low and high fluence samples (black open squares).

We first consider the results associated to the low energy implanted samples (crack depth position at $60 \mathrm{~nm}$ ). The values of $\Phi_{H_{2}}-\varphi_{H_{2}}^{\text {int.surf }}$ are much smaller than $\rho_{H_{2}}$ for both low and high fluence samples (Fig. 10, red solid squares). In other words, there are not enough hydrogen molecules available to pressurize the observed blisters. In addition, since we have shown that they cannot be generated only by $\mathrm{He}$ 
TABLE I. Areal densities of $\mathrm{He}$ atoms, $\varphi_{H e}$, and $\mathrm{H}_{2}$ molecules, $\varphi_{H_{2}}$, contained within the populations of blisters.

\begin{tabular}{|c|c|c|c|c|c|c|}
\hline \multirow[b]{2}{*}{ Molecule density $\left(\times 10^{16} \mathrm{~cm}^{-2}\right)$} & \multicolumn{2}{|c|}{ Low energy } & \multicolumn{2}{|c|}{ Moderate energy } & \multicolumn{2}{|c|}{ High energy } \\
\hline & $\varphi_{\mathrm{He}}$ & $\varphi_{\mathrm{H}_{2}}$ & $\varphi_{\mathrm{He}}$ & $\varphi_{\mathrm{H}_{2}}$ & $\varphi_{\mathrm{He}}$ & $\varphi_{\mathrm{H}_{2}}$ \\
\hline Low fluence & 0.6 & 0.2 & $(0.6-0.4) \mathbf{0 . 4}$ & $(0.1-0.2) \mathbf{0 . 2}$ & $(0.5-0.2) 0.3$ & $(0-0.2) \mathbf{0 . 1}$ \\
\hline High fluence & 1.2 & 0.5 & $(1.2-0.7) 0.9$ & $(0.1-0.5) \mathbf{0 . 4}$ & $(1.1-0.5) 0.7$ & $(0-0.5) 0.3$ \\
\hline
\end{tabular}

molecules, real blister cavities are necessarily pressurized by a mixture of $\mathrm{He}$ and $\mathrm{H}_{2}$ molecules.

More generally, the areal densities of He atoms, $\varphi_{H e}$, and $\mathrm{H}_{2}$ molecules, $\varphi_{\mathrm{H}_{2}}$, contained within the population of blister cavities formed at a depth of $60 \mathrm{~nm}$ should satisfy the conditions

$$
\left\{\begin{array}{l}
\varphi_{\mathrm{He}}+\varphi_{\mathrm{H}_{2}}=x \rho_{\mathrm{He}}+(1-x) \rho_{\mathrm{H}_{2}}, \\
x=\frac{\varphi_{\mathrm{He}}}{\varphi_{\mathrm{He}}+\varphi_{\mathrm{H}_{2}}},
\end{array}\right.
$$

that yields $\varphi_{H e}=1 / 2\left(\rho_{H e}-2 \varphi_{H_{2}} \pm \sqrt{\rho_{H e}^{2}+4 \varphi_{H_{2}}\left(\rho_{H_{2}}-\rho_{H e}\right)}\right)$. Taking into account that $\varphi_{H e} \leq \Phi_{H e}, \varphi_{H_{2}} \leq \Phi_{H_{2}}-\varphi_{H_{2}}^{\text {int.surf }}$ and substituting $\rho_{H e}$ and $\rho_{H_{2}}$ in Eq. (4) by their real values, we obtain $\varphi_{H e} \cong \Phi_{H e}, \varphi_{H_{2}} \cong \Phi_{H_{2}}-\varphi_{H_{2}}^{\text {int.surf }}$ and $x \cong 0.7$.

Thus, we demonstrate here that all the helium and the hydrogen ions implanted at low energy are contained within the populations of blister cavities formed by this implantation and annealing. This is an important result which shows that from the early precipitation of both gases in the form of complexes then their transformation into platelets and later on into cracks, this thermal evolution is conservative and thus that the proximities of the $\mathrm{Si} / \mathrm{SiO}_{2}$ interface and the surface of the wafer do not promote the exo-diffusion of any of the implanted gases.

An increase in the implantation energy results in a decrease of $\rho_{\mathrm{HeH}_{2}}$ whatever the value of $x$ (Fig. 10). Thus, the populations of blister cavities formed at $100 \mathrm{~nm}$ and $140 \mathrm{~nm}$ from the wafer surface should contain a smaller density of $\mathrm{He}$ and $\mathrm{H}_{2}$ molecules than available through the total implanted fluences. Using an approach similar to that applied above for the case of the low energy implanted sample, we can estimate the ranges of $\mathrm{He}$ and $\mathrm{H}_{2}$ molecule areal densities that contribute to pressurizing the observed blisters (Table I, boundary values are indicated in parentheses).

For all the implantation conditions studied in this work, helium always diffuses towards hydrogen to take part in the formation of platelets and cracks located on the $\mathrm{H}$ depth profile. Thus, in this region, the $\mathrm{He}$ to $\mathrm{H}$ concentration ratio always equals 1 independently of the implantation

TABLE II. Portions of $\mathrm{He}$ and $\mathrm{H}$ implanted fluences contained within the populations of blisters.

\begin{tabular}{|c|c|c|c|c|c|c|}
\hline \multirow[b]{2}{*}{$\varphi / \Phi(\%)$} & \multicolumn{2}{|c|}{ Low energy } & \multicolumn{2}{|c|}{ Moderate energy } & \multicolumn{2}{|c|}{ High energy } \\
\hline & $\mathrm{He}$ & $\mathrm{H}$ & $\mathrm{He}$ & $\mathrm{H}$ & $\mathrm{He}$ & $\mathrm{H}$ \\
\hline Low fluence & 100 & 100 & 70 & 95 & 50 & 59 \\
\hline High fluence & 100 & 100 & 75 & 82 & 58 & 67 \\
\hline
\end{tabular}

conditions. Bearing in mind that the pressure inside a blister cavity is independent of its depth and of the implanted fluence (Fig. 8), all the blisters are probably pressurized using the same gas mixture, whatever the implantation conditions. Otherwise, one would have to admit that there is an inhomogeneous lateral and/or depth distribution of $\mathrm{H}$ and $\mathrm{He}$ ions after implantation, which is highly unlikely. Since for the low energy ion implanted sample, we explicitly find this mixture ratio $x \cong 0.7$, we can estimate the areal densities of $\mathrm{H}_{2}$ and He molecules contained within the populations of blisters observed after moderate and high energy implantations. These estimations are shown in bold in Table I and fall well within the intervals proposed earlier in this paragraph. More generally, these data show that the higher the ion implantation energy is, the lower the areal densities of helium atoms and hydrogen molecules which pressurize the observed blisters.

Finally, we estimate the portions of implanted fluences which are found within the different populations of blister cavities, $\varphi_{H e} / \Phi_{H e}$ for $\mathrm{He}$ atoms and $2\left(\varphi_{H_{2}}+\varphi_{H_{2}}^{\text {int.surf }}\right) / \Phi_{H}$ for $\mathrm{H}$ atoms (Table II). These results show that when the implantation energy increases the portions of the $\mathrm{He}^{+}$and $\mathrm{H}^{+}$ implanted fluences finally found in the blisters decrease. This portion, being of $100 \%$ for the low energy implanted samples, decreases down to $(60 \pm 10) \%$ for the high energy implanted samples.

Since the thermal evolution of platelets and blisters has been proven to be conservative (no $\mathrm{H}$ or $\mathrm{He}$ losses) in the samples implanted close to the surface $(60 \mathrm{~nm}$ depth position), these evolutions must also be conservative when the cracks are located deeper. Thus, the rest of the $\mathrm{He}^{+}$and $\mathrm{H}^{+}$ implanted fluences must be found within "precipitates" other than blister cavities, in other words within the platelets. We have indeed observed that when increasing the implantation energy the population of platelets tends to distribute over wider depths. The cracks being formed within a relatively thin layer as a result of the mechanical coalescence of the platelets distributed at this depth, when the platelets are widely depth distributed, many of them, located outside this layer, are not involved in the formation of cracks and, then, blister cavities. This we have shown by TEM (see Fig. 2) after moderate and high energy implantations and well corroborate our conclusions.

\section{CONCLUSIONS}

In this work, we have studied the cracks and blisters that are formed following the co-implantation of $\mathrm{He}^{+}$then $\mathrm{H}^{+}$ ions at low energies and annealing under conditions close to those used at conventional energy ion implantation in the Smart Cut technology. We have shown that the blisters are 
formed due to the elastic deformation of the Si crystal close to the free surface of the wafer. During annealing, all the He atoms diffuse towards the region implanted with $\mathrm{H}^{+}$ions and participate to the formation and pressurization of platelets and blister cavities. Once a depth position of a crack is fixed, the height of a blister is only a function of its diameter. We have modelled by FEM the characteristics of blisters and used this model to infer the gas pressure within a blister cavity from a blister height measured experimentally. From there, we were able to demonstrate that the pressure within a blister cavity only depends on its diameter and not on other experimental conditions (energy, fluence). We have demonstrated that the pressure inside a blister located close to the wafer surface can be analytically calculated as the pressure inside a plate-like crack, embedded within an infinite medium, of the same diameter and containing the same gas concentration.

Comparing the gas densities needed to pressurize the observed blisters to the implanted fluences, we have shown that blister cavities cannot be pressurized by $\mathrm{H}_{2}$ only or $\mathrm{He}$ only molecules but by a gas mixture. We have estimated the $\mathrm{He} / \mathrm{H}_{2}$ ratio within the blisters and found that this mixture consists in approximately $70 \%$ of $\mathrm{He}$ and $30 \%$ of $\mathrm{H}_{2}$ molecules, the rest of hydrogen being used to passivate the internal surfaces of the blister cavities.

Moreover, after low-energy ion implantation and in contrast to what is assumed in the state of the art, all the implanted ions are gathered within the blister cavities, showing that no-exodiffusion occurs even when the cracks are located very close $(60 \mathrm{~nm})$ to the wafer surface and/or to some $\mathrm{SiO}_{2} / \mathrm{Si}$ interface. When the ion implantation energy increases, the platelets, from which the cracks are formed, become more widely distributed over different depths, and many of them survive the formation of cracks leading to a decrease in the portion of the implanted fluences which readily contribute to the pressurization of blister cavities. Thus, the failure of the conventional Smart Cut technology at low and very low ion energies cannot be ascribed to the exodiffusion of the implanted gases in the vicinity of the wafer surface. Alternative origins for this failure must be investigated.

${ }^{1}$ M. Bruel, Mater. Res. Innovations 3, 9 (1999).

${ }^{2}$ S. Pillai, K. R. Catchpole, T. Trupke, and M. A. Green, J. Appl. Phys. 101(9), 093105 (2007).

${ }^{3}$ O. Moutanabbir and U. Gösele, "Heterogeneous integration of compound semiconductors," in Annual Review of Materials Research, edited by D. R. Clarke, M. Rühle, and F. Zok (Annual Reviews, 2010), Vol. 40, pp. 469-500.

${ }^{4}$ J. Grisolia, G. B. Assayag, A. Claverie, B. Aspar, C. Lagahe, and L. Laanab, Appl. Phys. Lett. 76(7), 852 (2000).

${ }^{5}$ S. Personnic, K. K. Bourdelle, F. Letertre, A. Tauzin, N. Cherkashin, A. Claverie, R. Fortunier, and H. Klocker, J. Appl. Phys. 103, 023508 (2008).

${ }^{6}$ N. Daix, Ph.D. thesis, University Toulouse III-Paul Sabatier, 2009.

${ }^{7}$ N. Sugii, Microelectron. Eng. 132, 226-235 (2015).

${ }^{8}$ C. Qian and B. Terreault, J. Appl. Phys. 90, 5152 (2001).

${ }^{9}$ O. Moutanabbir and B. Terreault, Appl. Phys. Lett. 86, 051906 (2005).

${ }^{10}$ N. Cherkashin, F.-X. Darras, P. Pochet, S. Reboh, N. Ratel-Ramond, and A. Claverie, Acta Mater. 99, 187-195 (2015).

${ }^{11}$ O. Moutanabbir, B. Terreault, M. Chicoine, F. Schiettekatte, and P. J. Simpson, Phys. Rev. B 75, 075201 (2007).

${ }^{12}$ A. Agarwal, T. E. Haynes, V. C. Venezia, O. W. Holland, and D. J. Eaglesham, Appl. Phys. Lett. 72(9), 1086 (1998).

${ }^{13}$ J.-D. Penot, D. Massy, F. Rieutord, F. Mazen, S. Reboh, F. Madeira, L. Capello, D. Landru, and O. Kononchuk, J. Appl. Phys. 114, 123513 (2013).

${ }^{14}$ X. Hebras, P. Nguyen, K. K. Bourdelle, F. Letertre, N. Cherkashin, and A. Claverie, Nucl. Instrum. Methods Phys. Res., Sect. B 262, 24 (2007).

${ }^{15} \mathrm{~N}$. Cherkashin and A. Claverie, "Characterization of process-induced defects," in TEM in Micro-nanoelectronics, edited by A. Claverie (Wiley, 2012), pp. 165-193, ISBN: 9781848213678.

${ }^{16}$ J. F. Ziegler and J. P. Biersack, "SRIM computer code," see http:// www.srim.org.

${ }^{17}$ N. Enomoto, S. Muto, T. Tanabe, J. W. Davis, and A. A. Haasz, J. Nucl. Mater. 385, 606-614 (2009)

${ }^{18}$ G. Parry, C. Coupeau, E. Dion, M.-L. David, J. Colin, and J. Grilhé, J. Appl. Phys. 110, 114903 (2011).

${ }^{19}$ C. Coupeau, G. Parry, J. Colin, M.-L. David, J. Labanowski, and J. Grilhé, Appl. Phys. Lett. 103, 031908 (2013).

${ }^{20}$ J. Grisolia, G. B. Assayag, B. de Mauduit, A. Claverie, R. E. Kroon, and J. H. Neethling, MRS Proc. 681, I3.2 (2001).

${ }^{21}$ S. Muto, S. Takeda, and M. Hirata, Philos. Mag. A 72(4), 1057-1074 (1995).

${ }^{22}$ M. Lallemand and D. Vidal, J. Chem. Phys. 66, 4776 (1977).

${ }^{23}$ H. Shimizu, E. M. Brody, H. K. Mao, and P. M. Bell, Phys. Rev. Lett. 47, 128 (1981).

${ }^{24}$ C. G. Van De Walle and R. Street, Phys. Rev. B 51(16), 10615 (1995). 